\title{
Secondary Nicotinic Synapses on Sympathetic B Neurons and Their Putative Role in Ganglionic Amplification of Activity
}

\author{
Paul Karila and John P. Horn \\ Department of Neurobiology, University of Pittsburgh, School of Medicine, Pittsburgh, Pennsylvania 15261
}

The strength and number of nicotinic synapses that converge on secretomotor $B$ neurons were assessed in the bullfrog by recording intracellularly from isolated preparations of paravertebral sympathetic ganglia 9 and 10. One input to every B neuron invariably produced a suprathreshold EPSP and was defined as the primary nicotinic synapse. In addition, 93\% of the cells received one to four subthreshold inputs that were defined as secondary nicotinic synapses. This contradicts the prevailing view, which has long held that amphibian B neurons are singly innervated. More important, the results revealed that B cells provide the simplest possible experimental system for examining the role of secondary nicotinic synapses on sympathetic neurons. Combining the convergence data with previous estimates of divergence indicates that the average preganglionic B neuron forms connections with 50 ganglionic $B$ neurons and that the majority of these nicotinic synapses are secondary in strength. Secondary EPSPs evoked by low-frequency stimulation ranged from 0.5 to $10 \mathrm{mV}$ in amplitude and had an average quantal content of 1 . Nonetheless, secondary synapses could trigger action potentials via four mechanisms: spontaneous fluctuations of EPSP amplitude, two-pulse facilitation, coactivation with other secondary synapses, and coactivation with a slow peptidergic EPSP. The data were used to formulate a stochastic theory of integration, which predicts that ganglia function as amplifiers of the sympathetic outflow. In this two-component scheme, primary nicotinic synapses mediate invariant synaptic gain, and secondary nicotinic synapses mediate activity-dependent synaptic gain. The model also provides a common framework for considering how facilitation, metabotropic mechanisms, and preganglionic oscillators regulate synaptic amplification in sympathetic ganglia.

Key words: activity-dependent modulation; bullfrog sympathetic ganglia; neuronal nicotinic receptors; nicotinic synapses; metabotropic synapses; synaptic integration; sympathetic nervous system
Synaptic convergence is a basic determinant of neuronal integration, and it appears to follow a simple pattern in paravertebral sympathetic ganglia. Sympathetic neurons are generally innervated by one preganglionic axon that forms a strong nicotinic synapse and by a variable number of axons that form weaker nicotinic synapses (Dodd and Horn, 1983b; Skok and Ivanov, 1983; Hirst and McLachlan, 1986; Jänig and McLachlan, 1992). In this paper, we define nicotinic synapses as primary when they produce EPSPs that are always suprathreshold in strength and as secondary when they produce EPSPs that are generally subthreshold during low-frequency stimulation. Variability in the number of secondary synapses per neuron occurs within ganglia and between species. The precise physiological role of ganglionic convergence and its variation remain as an interesting unsolved problem.

One approach to the problem of convergence has stressed the developmental mechanisms that specify formation of appropriate

\footnotetext{
Received June 7, 1999; revised Nov. 5, 1999; accepted Nov. 11, 1999.

This work was supported by a postdoctoral fellowship to P.K. from the Swedish Foundation for International Cooperation in Research and Higher Education (Stiftelsen för Internationalisering au Högre Utbildning och Forskning) and by National Institutes of Health Grant NS21065 to J.P.H. We thank Eric Frank for advice about quantal analysis, and we are grateful to Elias Aizenman, Jed Hartings, Hermann Schobesberger, and the anonymous reviewers for many helpful comments.

Correspondence should be addressed to Dr. John P. Horn, Department of Neurobiology, University of Pittsburgh, School of Medicine, E1440 Biomedical Science Tower, Pittsburgh, PA 15261. E-mail: jph+@pitt.edu.

Dr. Karila's present address: Preclinical Development, B209:2, AstraZeneca R\&D Södertälje, SE-151 85 Södertälje, Sweden.

Copyright (C) 2000 Society for Neuroscience $\quad 0270-6474 / 00 / 200908-11 \$ 15.00 / 0$
}

synaptic connections between preganglionic and postganglionic neurons. Comparison of the superior cervical ganglion (SCG) in five mammalian species revealed that preganglionic to postganglionic convergence ranged from 4 to 15 and correlated with the number of primary dendrites on postganglionic neurons (Purves and Lichtman, 1985). Although the significance of this arrangement remains unknown, this work also uncovered an interesting correlation between the body weight of a species and the average number of dendrites on sympathetic neurons (Purves et al., 1986). As a possible explanation, it was proposed that developmental regulation of convergence produces some kind of scaling effect on sympathetic function in animals of different sizes.

Our approach to convergence focuses on its integrative consequences in functional subsets of sympathetic neurons, using anuran amphibians as the model. Convergence in paravertebral ganglia 9 and 10 of frogs and toads is lower than that in the mammalian SCG and appears to differ between secretomotor B neurons and vasomotor $\mathrm{C}$ neurons. $\mathrm{B}$ cells, which innervate cutaneous glands (Lang et al., 1975; Horn et al., 1988; Jobling and Horn, 1996), may exemplify the simplest possible system. In the original identification of the $\mathrm{B}$ and $\mathrm{C}$ cell types, it was found that virtually all B neurons are innervated by only one axon, which forms a primary synapse (Nishi et al., 1965). This widely accepted view has been consistently supported in anecdotal reports (Blackman et al., 1963a; Skok, 1973; Weitsen and Weight, 1977; Dodd and Horn, 1983a). By comparison, it is relatively easy to demonstrate one to three secondary synapses on most vasomotor $\mathrm{C}$ neurons (Dodd and Horn, 1983b). The apparent difference suggests that secondary nicotinic synapses contribute to the inte- 
A

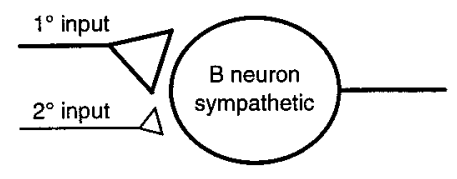

B

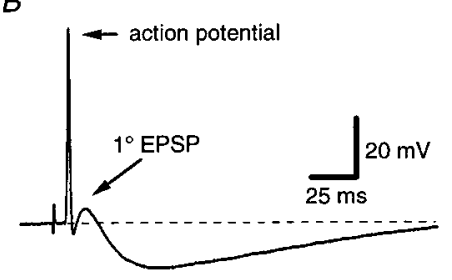

$c$

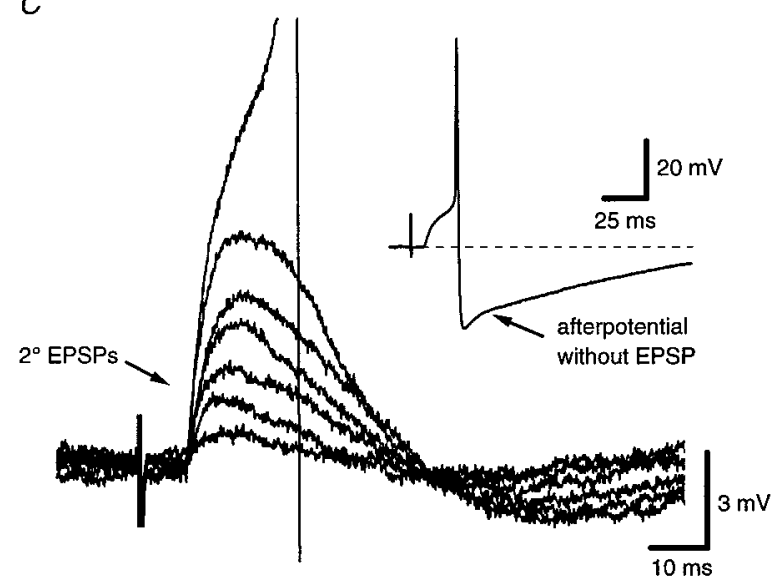

Figure 1. Identification of primary and secondary nicotinic EPSPs. $A$, Schematic for convergence of primary $\left(1^{\circ}\right)$ and secondary $\left(2^{\circ}\right)$ synapses on a sympathetic B neuron is shown. $B$, Presynaptic stimulation of the primary synapse evoked an invariably suprathreshold EPSP, which distorted the action potential afterhyperpolarization. $C$, Lowering presynaptic stimulus strength revealed subthreshold secondary EPSPs. Superimposed records illustrate the range of spontaneous fluctuations in EPSP amplitude. In this cell, the secondary EPSP occasionally crossed threshold. Inset, Spike afterpotentials triggered by the secondary synapse showed little sign of an EPSP. $\mathrm{V}_{m}=-48 \mathrm{mV}$. grated output of action potentials by $\mathrm{C}$ neurons but not $\mathrm{B}$ neurons.

We decided to examine the issue of secondary nicotinic synapses after Ivanoff and Smith (1995) observed subthreshold nicotinic EPSPs in $53 \%$ of B cells during spontaneous activity in vivo. This surprising finding countered all previous reports and led the authors to propose that a novel contralateral preganglionic pathway is cut when ganglia are isolated for study in vitro. The initial aims of the present work were to clarify the extent of polyinnervation in B neurons and to test its impact on postsynaptic firing of action potentials. The results led us to formulate a general theory of ganglionic integration.

\section{MATERIALS AND METHODS}

Twenty-eight bullfrogs (Rana catesbeiana; $14-18 \mathrm{~cm}$ ) of both sexes were cooled on ice for $30 \mathrm{~min}$ and killed by double pithing. Unilateral preparations of the paravertebral chain, including ganglia 7-10 and the associated spinal nerves, were isolated and pinned flat in a recording dish (Dodd and Horn, 1983a). In this preparation, graded preganglionic stimulation allows for the fractionation and characterization of nicotinic synapses because all bullfrog sympathetic neurons have a catecholaminergic phenotype (Stofer and Horn, 1990) and receive their cholinergic innervation from preganglionic neurons in the spinal cord (Horn and Stofer, 1988; Smith, 1994). Suction electrodes were fitted on the chain above ganglion 7 for stimulation of the presynaptic B pathway and on spinal nerves 7 and 8 for separate stimulation of the presynaptic $\mathrm{C}$ pathway (Dodd and Horn, 1983a). Having electrodes on both preganglionic pathways aided cell identification, and in some experiments (e.g., see Fig. 7) the C pathway was stimulated to evoke a peptidergic EPSP in B neurons (Jan et al., 1979). Preparations were superfused $\left(1 \mathrm{ml} \mathrm{min}{ }^{-1}\right)$ at room temperature $\left(20-22^{\circ} \mathrm{C}\right)$ with Ringer's solution $(\mathrm{mM})$ : $115 \mathrm{NaCl}, 2$ $\mathrm{KCl}, 1.8 \mathrm{CaCl}_{2}$, and 4 HEPES, $\mathrm{pH}$ 7.2.

Intracellular recording. Neurons were impaled under visual guidance $(40 \times$ water; Zeiss WL) with sharp microelectrodes filled with $3 \mathrm{M} \mathrm{KCl}$ (70-90 $\mathrm{M} \Omega$ ). In some experiments, electrodes were beveled to lower their resistance (15-35 M $\Omega$ ). B neurons in ganglia 9 and 10 were identified by the segmental origin and conduction velocities of their nicotinic inputs (Dodd and Horn, 1983a). Recordings were monitored on an oscilloscope and chart recorder and digitized at $10 \mathrm{kHz}$. After impalement, cells were allowed to stabilize for $>5$ min before data collection. General analysis and graphing were performed with IGOR Pro 3.12 for Windows (WaveMetrics, Lake Oswego, OR). Grouped data are expressed as the mean \pm SEM.

During characterization of convergence, cellular leak resistance $\left(R_{\text {leak }}\right)$ was measured at regular intervals and just before pulling out, when possible. To estimate $R_{\text {leak }}, I-V$ curves were constructed, typically using $500 \mathrm{msec}$ current pulses with $50 \mathrm{pA}$ steps. After subtracting unbalanced electrode resistance, $R_{\text {leak }}$ was taken as the slope of the $I-V$ relation in the linear range between -60 and $-110 \mathrm{mV}$. This provides an index of impalement damage (Jones, 1989).

Counting of nicotinic synapses. Synaptic inputs to B neurons were fractionated by varying the presynaptic stimulus intensity. Lowfrequency stimulation $(0.2 \mathrm{~Hz})$ was used to avoid effects of facilitation and depression (Shen and Horn, 1995). Stimulus parameters of 0.1-0.4 msec and $0.5-2.5 \mathrm{~V}$ produced the best resolution between individual presynaptic axons. In counting synapses, we varied the stimulus intensity and looked for clearly discernible steps in the average EPSP amplitude or shape, arising from different conduction velocities of newly recruited inputs. Latency shifts were never used as the sole criterion because latency can vary with stimulus intensity, because of current spread (Lichtman, 1980). This method of graded stimulation provides a minimal estimate of convergence because it only detects those secondary synapses whose presynaptic stimulus thresholds are lower than that of the primary presynaptic axon.

Quantal analysis of secondary nicotinic synapses. Stimulus strength was adjusted to minimize the rate of transmission failure during selective activation of a single preganglionic axon (Allen and Stevens, 1994; Dobrunz and Stevens, 1997). In seven such neurons, asynchronous EPSPs were also observed and subsequently analyzed using an event detection program [AxoGraph 3.5; Axon Instruments (Clements and Bekkers, 1997)]. The mean amplitudes of asynchronous EPSPs $(x)$ and evoked EPSPs $(X)$ were used for direct, model-independent, calculation of quantal content $\left(m_{\text {direct }}=X[x]^{-1}\right)$. The probability of release $(p)$ was then estimated by fitting the data to a binomial distribution and assuming different numbers of release sites $(N)$. The best fit was chosen by $\chi^{2}$ statistics and accepted when the probability of its occurrence by chance was $<0.05$. For purposes of minimal stimulation and illustration (e.g., see Fig. 2), transmission failures were assessed by visual inspection of records. Estimates of $X$ for quantal analysis include data from all stimulus trials. Elsewhere in the results, visually discriminated failures were removed from the data before calculating average secondary EPSP amplitudes.

\section{RESULTS}

\section{Distribution of secondary nicotinic synapses}

We begin by presenting examples of convergence and follow with grouped data. The first case illustrates a B neuron with two synaptic inputs (Fig. 1A). Supramaximal stimulation of the preganglionic B pathway evoked a primary EPSP that invariably triggered an action potential (Fig. $1 B$ ). During recovery of the action potential, the initial phase of the spike afterhyperpolarization was clearly distorted by the fast EPSP. This profile matches the classical picture of mononeuronal innervation by a single strong nicotinic synapse (Blackman et al., 1963a; Nishi et al., 1965). In a departure from the accepted view, we observed a subthreshold secondary EPSP after reducing the supramaximal stimulus (Fig. 1C). In this particular cell one could go back and forth repeatedly between the primary and secondary inputs for $>1$ hr by simply adjusting the stimulus intensity, thus showing it is possible to isolate and activate selectively a secondary synapse. 
$A$
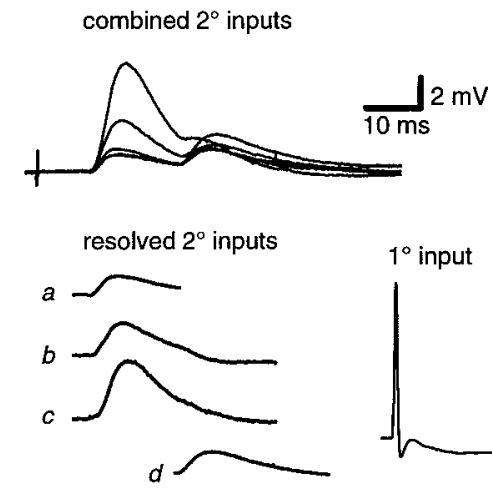

$1^{\circ}$ input

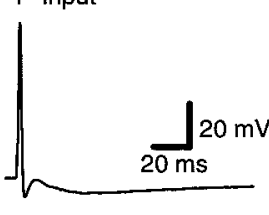

C

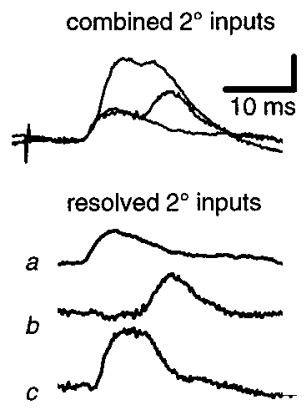

$B$
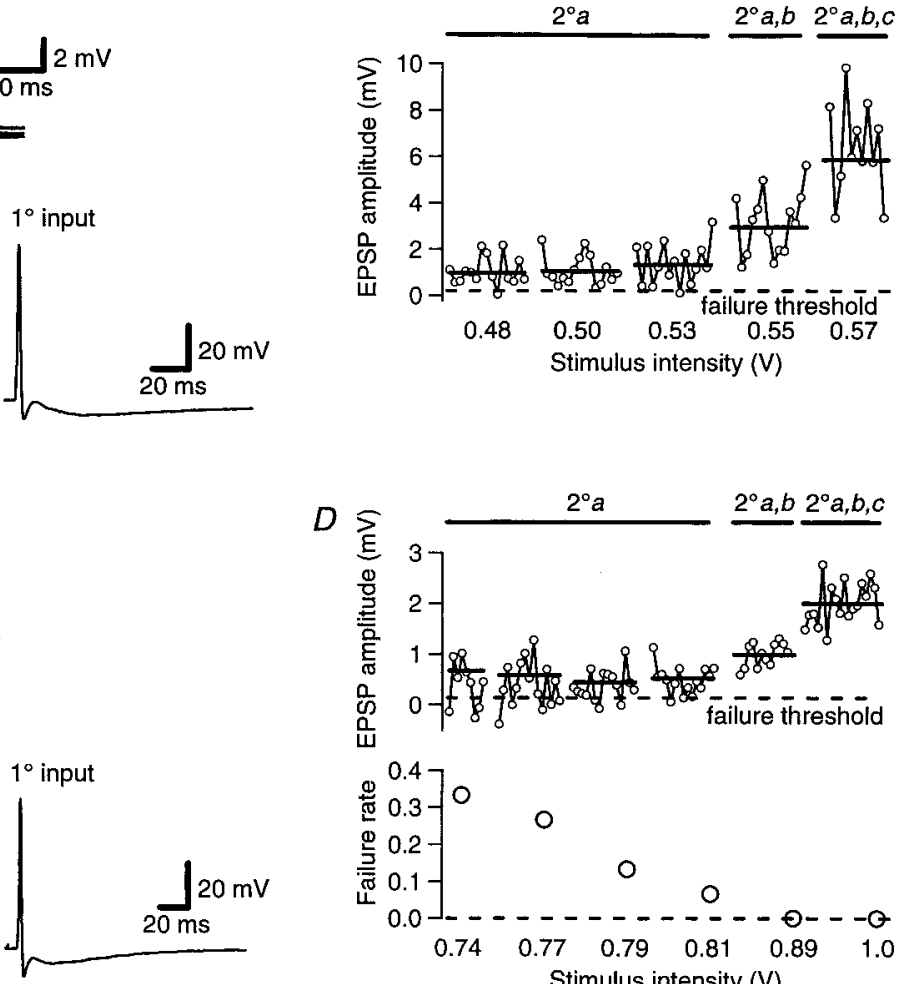

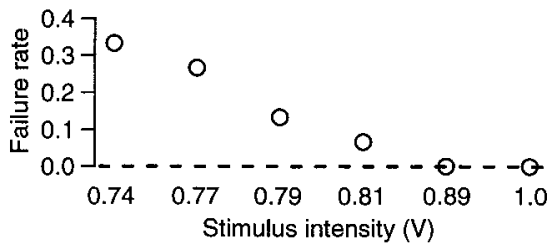

Figure 2. Examples of two B neurons with relatively high levels of synaptic convergence. One cell had five synapses $(A, B)$, and the other had four synapses $(C, D) . A, C$, Combined and resolved secondary EPSPs from each cell are depicted. Also shown are action potentials $($ right $)$ initiated by the primary synapses. Each record is an average of 9-18 responses at $0.2 \mathrm{~Hz}$. Individual components of the combined EPSP each had a distinct stimulus threshold or latency. Components of secondary EPSPs were resolved by subtraction. For example, input $a$ in $A$ was selectively evoked by stimuli of $0.48-0.53 \mathrm{~V}$, inputs $a+b$ were evoked by $0.55 \mathrm{~V}$ stimuli, and input $b$ was resolved by subtracting $a$ from $a+b$. $B, D$, Top, The relation between stimulus intensity and secondary EPSP amplitude illustrates the differences in threshold for individual secondary synapses. $D$, Bottom, The transmission failure rate decreased as stimulus intensity was increased.

During 600 trials at $0.25 \mathrm{~Hz}$, the amplitude of the secondary EPSP fluctuated between 1 and $10 \mathrm{mV}$, and it occasionally crossed threshold (Fig. 1C). However, unlike the primary EPSP, the much weaker secondary EPSP produced little effect on the spike afterpotential (Fig. 1C, inset). In those stimulus trials in which the secondary EPSP approached threshold, it clearly activated voltage-dependent currents, as evidenced by a prolonged peak, an accelerated decay, and an undershoot (Fig. 1C).

Two examples of B neurons with higher levels of convergence are illustrated in Figure 2. In both cases, graded increases in presynaptic stimulus strength revealed multiple steps in the average EPSP amplitude and finally recruited a primary synapse that triggered an action potential. The neuron in Figure 2, $A$ and $B$, had five inputs, the most observed in this study. They consisted of one primary and four secondary synapses. Three of the secondary EPSPs had similar latencies but differed in their average amplitudes and stimulus thresholds (Fig. 2A,B). The fourth secondary synapse was distinguished by its longer latency. Our final example is from a neuron with four inputs (Fig. $2 C, D$ ). In this case, the synapse labeled $a$ had the lowest stimulus threshold, and its failure rate declined as stimulus intensity was increased (Fig. $2 C, D)$. Adjusting the stimulus strength in this manner exemplifies the technique of minimal stimulation, which was used to minimize presynaptic action potential failures in subsequent release experiments. In cells with multiple secondary synapses, we attempted to resolve the time course and amplitude of individual components by subtracting averaged EPSPs representing different combinations of inputs (Fig. 2A,C). This approach will work when EPSP amplitudes are much smaller than the driving force on synaptic currents and summation is linear. However, subtraction revealed evidence in some cells of EPSPs with rounded peaks (Fig. $2 C$, input $c$ ) and the other signs of voltage-dependent currents (e.g., Fig. $1 C$ ). Because of the activation of these nonsynaptic currents, summation is likely to be nonlinear. Thus the subtraction approach provides only an approximate estimate of secondary EPSP components, and it could not be used to quantitate the size of individual responses.

The distribution of primary and secondary nicotinic synapses was analyzed in two groups of B neurons. In the first group (67 neurons; 14 frogs), the goal was to minimize sampling bias by including all neurons that were innervated and could generate action potentials $>45 \mathrm{mV}$. All 67 neurons had one primary nicotinic synapse, $93 \%$ had at least one secondary nicotinic synapse, and $22 \%$ had two or three secondary synapses. On average, $2.2 \pm 0.1$ axons converged onto each neuron, and secondary EPSP amplitude (excluding failures) was $2.6 \pm 0.3 \mathrm{mV}(n=37)$. For cells with $>1$ secondary input, only the EPSP with the lowest stimulus threshold was included in the averaged data. In this group, the resting membrane potential $\left(\mathrm{V}_{m}\right)$ was $-48 \pm 1 \mathrm{mV}$ $(n=55), R_{\text {leak }}$ was $133 \pm 21 \mathrm{M} \Omega(n=43)$, and the action potential threshold was $-33 \pm 2 \mathrm{mV}(n=43)$. The number of inputs per cell was independent of the quality of recordings, as 
$A$

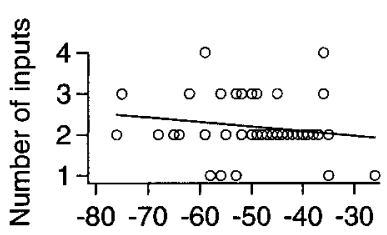

$B$
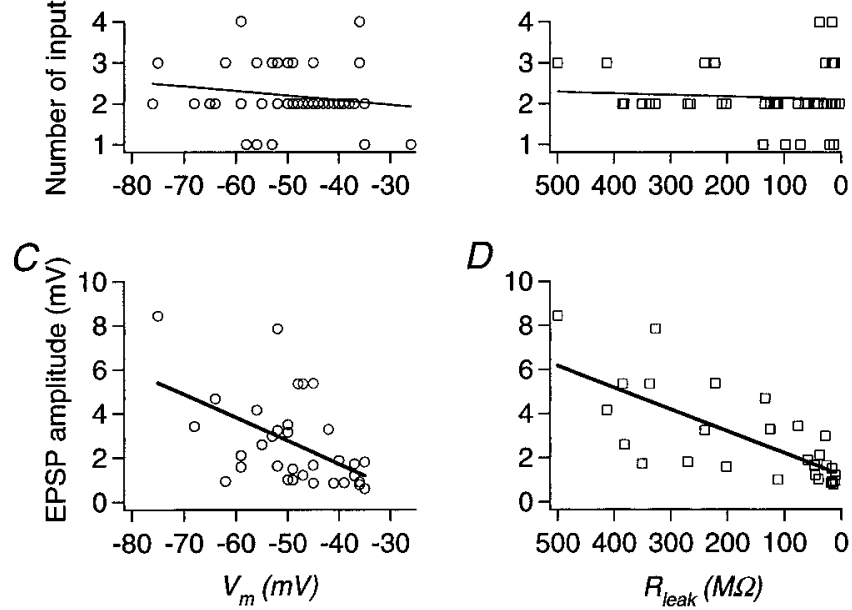

$D$

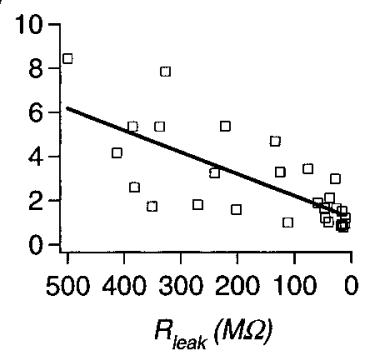

Figure 3. Impact of resting membrane properties on estimates of convergence and secondary EPSP amplitude. $A, B$, The number of secondary nicotinic synapses per neuron did not correlate with $\mathrm{V}_{m}(A)$ and $R_{\text {leak }}(B)$. $C, D$, Secondary EPSP amplitude declined with decreases in $\mathrm{V}_{m}(C)$ and $R_{\text {leak }}(D)$. Each point $[\bigcirc(A, C) ; \square(B, D)]$ in the scatter plots represents data from one cell. Lines are drawn by linear regression.

measured by $\mathrm{V}_{m}$ or $R_{\text {leak }}$ (Fig. $3 A, B$ ). This is important because it argues against the possibility that recording damage obscured secondary EPSPs in previous studies of B neurons. As would be expected from consideration of the driving force on synaptic currents and the shunting influence of the nonsynaptic membrane resistance, EPSP amplitudes were larger in cells with higher $\mathrm{V}_{m}$ and $R_{\text {leak }}$ (Fig. 3C,D).

In a second group of 32 neurons from 14 additional frogs, recordings were made using lower resistance beveled electrodes to reduce noise, and cells without well isolated secondary synapses were discarded. This group was similar to the first in that all cells received one primary synapse, and $\mathrm{V}_{m}=-48 \pm 1 \mathrm{mV}(n=$ 32). As would be expected from the more stringent selection criteria and the coarser electrodes, cells in the second group were characterized by higher convergence $(2.7 \pm 0.1$ inputs per cell; $n=32)$, lower $R_{\text {leak }}(27 \pm 4 \mathrm{M} \Omega ; n=22)$, and lower secondary EPSP amplitude (1.8. $\pm 0.3 \mathrm{mV} ; n=32)$. This group contained the cell with five inputs (Fig. $2 A, B$ ).

Overall, the pattern of convergence that we observed in sympathetic B neurons was reminiscent of previous work on parasympathetic neurons in the frog cardiac ganglion, where $55 \%$ of the cells receive one to four secondary synapses in addition to a primary input (Dennis and Sargent, 1978; Ko and Roper, 1978). In subsequent experiments, we attempted to assess the function of secondary synapses.

\section{Quantal properties of secondary nicotinic synapses}

Quantal content $(m)$ and other release parameters provide valuable indices of synaptic function because they reflect synaptic strength and structure and they influence release dynamics. We therefore sought to measure the resting value of $m$ during lowfrequency stimulation in physiological $\left[\mathrm{Ca}^{2+}\right]$ and to determine whether simple Poisson or binomial models could describe secondary EPSP amplitudes. Although spontaneous acetylcholine release is rare in sympathetic ganglia, we found seven neurons in which nerve stimulation evoked a secondary EPSP and asynchronous EPSPs (e.g., Fig. 4A). The average amplitudes of these events were similar $(X=0.99 \pm 0.32 \mathrm{mV} ; x=1.00 \pm 0.13 \mathrm{mV})$.
By making the assumption that the magnitudes of asynchronous EPSPs reflected quantal size at secondary synaptic release sites we could calculate quantal content for each cell by the direct method ( $m=1.04 \pm 0.31$; range, $0.31-2.33)$. This sample of neurons was characterized by $2.7 \pm 0.2$ inputs per cell, $\mathrm{V}_{m}=$ $-50 \pm 2 \mathrm{mV}$, and $R_{\text {leak }}=17 \pm 4 \mathrm{M} \Omega$.

Estimating the probability of release $(p)$ and the number of release sites $(N)$ from distributions of EPSP amplitudes proved difficult. Figure $4 A$ illustrates a series of trials in which most presynaptic nerve stimuli evoked a short-latency EPSP and also a longer-latency asynchronous EPSP. These examples are from the cell in which we observed the largest number of asynchronous events (154). In this instance, the amplitude distribution of asynchronous EPSPs had a clear positive skew (Fig. 4B). Unfortunately, asynchronous EPSPs were too infrequent to assess their amplitude distributions in the other six cells in which they were observed. In another approach, $2 \mathrm{~mm} \mathrm{Sr}^{2+}$ was added during recordings from seven other $B$ neurons (data not shown) to elevate the rate of asynchronous release during stimulation of a secondary synapse (average number of events $=320 \pm 124$ ). In every one of these cells, the amplitude distribution of asynchronous EPSPs had a positive skew similar to that seen for asynchronous events in normal Ringer's solution. Skewed distributions like those in Figure $4 B$ and in the $\mathrm{Sr}^{2+}$-treated neurons are better described by a $\Gamma$ function than a Gaussian function. This resembles previous descriptions of spontaneous release in this preparation (Blackman et al., 1963b) and in other autonomic ganglia (Martin and Pilar, 1964; Dennis et al., 1971; McLachlan, 1975).

Histograms of synchronously evoked EPSP amplitudes were generally symmetric, without any sign of quantal peaks (Fig. $4 C, D)$. The absence of peaks presumably reflects a smearing effect produced by the quantum's high coefficient of variation (Fig. $4 B$ ), a by-product of the $\Gamma$ distribution. In theory a symmetric distribution of evoked EPSP amplitudes could arise from a Poisson process if $m$ were high (i.e., $>1$ ) or from a binomial model with intermediate values of $p$ (McLachlan, 1978). Overall, the data were not described by a Poisson distribution, and we were only successful (Fig. $4 D$ ) in fitting our data to a binomial model $(N=4 ; p=0.56)$ in one cell. As would be expected from a good fit, multiplication in this case of $N$ by $p$ to obtain $m$ (2.24) reproduced $m_{\text {direct }}(2.33)$.

\section{Excitatory action of secondary nicotinic synapses}

The physiological impact of secondary nicotinic synapses depends critically on their ability to initiate action potentials. In 5 of $99 \mathrm{~B}$ neurons, spontaneous fluctuations of secondary EPSP amplitude during $0.2 \mathrm{~Hz}$ stimulation were sufficient on their own to cause firing (Fig. 1C). To examine further the excitatory role of secondary synapses, we measured the consequences of repetitive stimulation and summation.

Pairing of stimulus shocks at intervals $<100$ msec consistently facilitated secondary EPSP amplitude and reduced the rate of transmission failures, when tested in 18 neurons. Figure $5 A$ illustrates the facilitation of subthreshold EPSP amplitude associated with interstimulus intervals of 10,30 , and $80 \mathrm{msec}$ (repetition rate $=0.1 \mathrm{~Hz}$ ). In grouped data (Fig. $5 B$ ), facilitation produced a maximal 3.2-fold increase in EPSP amplitude when stimuli were paired at $10 \mathrm{msec}$, the shortest interval examined.

Facilitation at a single secondary synapse also increased the probability of postsynaptic firing. In the case illustrated in Figure $5 A$, the first EPSP in each pair was always subthreshold. Delivery of the second stimulus $10 \mathrm{msec}$ later evoked an action potential in 

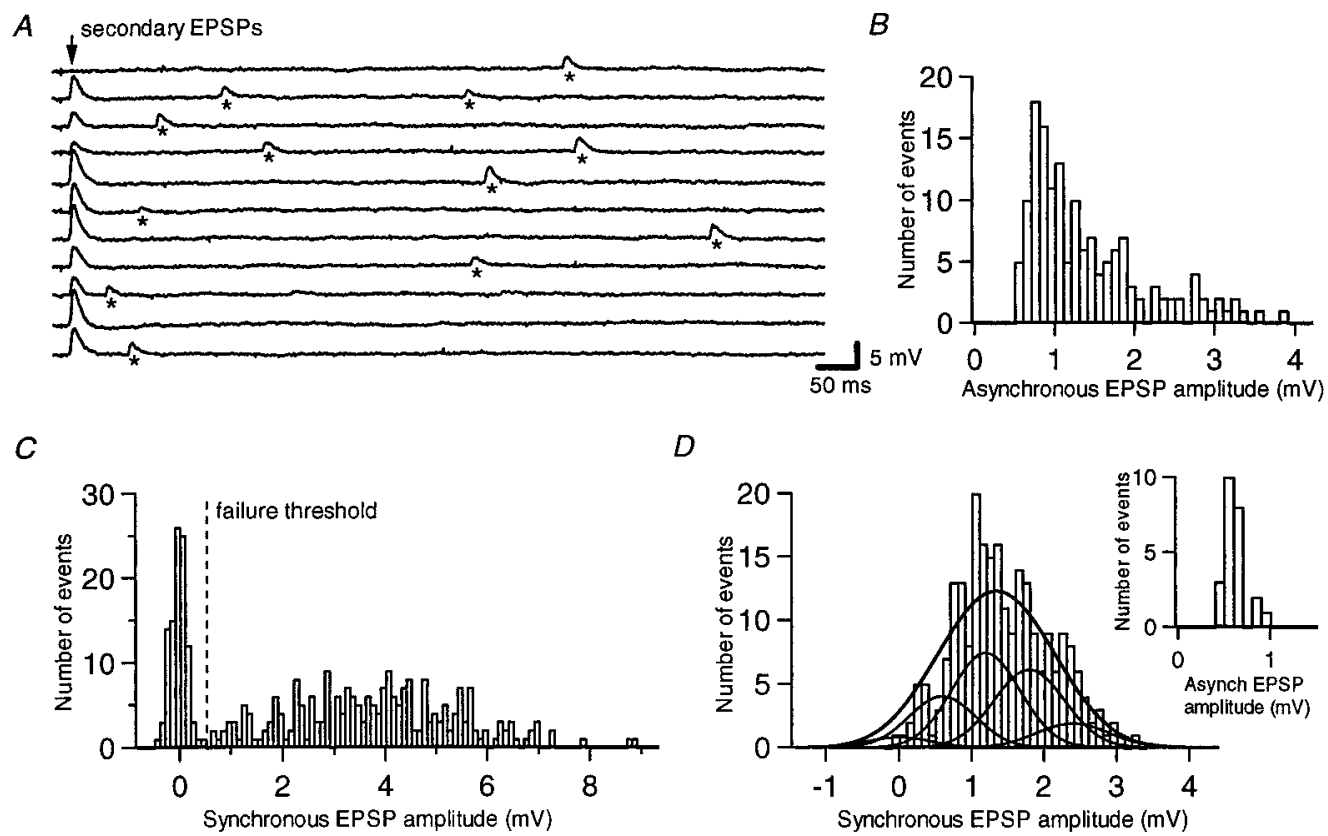

Figure 4. Analysis of secondary EPSP amplitudes. $A$, Selected stimulus trials illustrate EPSPs evoked by $0.2 \mathrm{~Hz}$ stimulation. In most of the trials shown, each nerve stimulus evoked a short-latency synchronous EPSP and an asynchronous EPSP (*) whose latency was longer and variable. An arrow in the first trial marks a failure of synchronous transmission. $B$, In an amplitude histogram, the distribution of 154 asynchronous EPSPs recorded from the neuron in $A$ shows a positive skew and an average amplitude $(x)$ of $1.39 \pm 0.02 \mathrm{mV}$. $C$, The amplitude histogram of synchronous EPSPs evoked from the same neuron $(X=2.67 \pm 0.13 ; 341$ trials) was broad and lacked discrete peaks at intervals corresponding to the average amplitude of asynchronous EPSPs. In this histogram, the peak near $0 \mathrm{mV}$ corresponds to 131 transmission failures. $D$, The distribution of EPSP amplitudes from another B neuron in which synchronous responses (large graph) and asynchronous responses (Asynch; inset) were recorded. The thick solid line in the large graph was drawn from a binomial fit in which $N=4$ and $p=0.56$. In this cell, $x=0.6 \pm 0.02 \mathrm{mV}(n=24)$, and $X=1.4 \pm 0.04 \mathrm{mV}(n=240)$. Thin lines in the histogram were generated by the binomial-fitting procedure, and they represent baseline noise and the expected distribution of EPSP amplitudes attributable to zero to four quantal events. Negative values in the overall fit (thick line) were introduced by the baseline noise.

11 of 20 trials. The excitatory efficacy of the second EPSP declined in this cell to $20 \%$ at $30 \mathrm{msec}$ and $0 \%$ at $80 \mathrm{msec}$. Facilitation may also operate over longer intervals to enhance postsynaptic firing. Figure $5 C$ shows 15 trials from another cell in which the first EPSP of the pair triggered 1 action potential and the second EPSP, $80 \mathrm{msec}$ later, triggered 10 action potentials. In general, the relation between the interstimulus interval and enhanced firing was quite variable between cells. The most effective interval ranged from 10 to $100 \mathrm{msec}$ in the four cells compared in Figure $5 D$. In seven cells in which $0.2 \mathrm{~Hz}$ stimulation of a secondary synapse never initiated firing, pairing stimuli at a 20 msec interval caused the second EPSP to trigger action potentials in $29.0 \pm 5.7 \%$ of trials. In this group, $\mathrm{V}_{m}=-50 \pm 3 \mathrm{mV}, R_{\text {leak }}$ $=112 \pm 48 \mathrm{M} \Omega$, number of inputs $=2.9 \pm 0.5$, and EPSP amplitude $=2.8 \pm 0.9 \mathrm{mV}$.

Coactivation of two convergent secondary synapses was also found to trigger action potentials in B neurons (Fig. 6). In a group of nine cells, summation of two secondary EPSPs stimulated action potentials in $21.3 \pm 2.8 \%$ of trials, whereas activation of one input produced action potentials in only $0.5 \pm 0.5 \%$ of trials. In this group $\mathrm{V}_{m}=-50 \pm 4 \mathrm{mV}, R_{\text {leak }}=121 \pm 61 \mathrm{M} \Omega$, number of inputs $=3.1 \pm 0.1$, and EPSP amplitude $=2.9 \pm 0.8 \mathrm{mV}$.

In the bullfrog, repetitive stimulation of the $\mathrm{C}$ pathway releases a luteinizing hormone-releasing hormone-like peptide, which diffuses to nearby B neurons and produces a slow EPSP lasting several minutes (Jan et al., 1979). Interaction between this slow metabotropic EPSP and secondary nicotinic EPSPs was complex. In eight neurons, we found that stimulation of the peptidergic EPSP (100 stimuli at $20 \mathrm{~Hz}$ ) inhibited transmission at secondary nicotinic synapses. The inhibition was not studied further but may arise from peptidergic inhibition of nicotinic receptors (Akasu et al., 1983). In six other B neurons, interaction between the fast and slow EPSPs clearly enhanced the firing of action potentials $\left(\mathrm{V}_{m}=\right.$ $-51 \pm 6 \mathrm{mV} ; R_{\text {leak }}=144 \pm 82 \mathrm{M} \Omega$; number of inputs $=3.2 \pm$ 0.5 ; EPSP amplitude $=2.8 \pm 1.1 \mathrm{mV}$ ). In control trials before peptide release, only $7.7 \pm 4.9 \%$ of fast EPSPs triggered action potentials. During slow EPSPs $(100$ stimuli at $20 \mathrm{~Hz})$, the proportion of suprathreshold nicotinic EPSPs increased to $16.7 \pm$ $7.1 \%$ ( $p \leq 0.05$, two-tailed paired Student's $t$ test). An example of the effect is shown in Figure $7 A$. It is interesting to note that some fast EPSPs were also inhibited in this experiment (Fig. $7 B$ ). This suggests that an inhibitory effect on the fast EPSP was again present, despite the fact that interaction with the peptidergic EPSP produced a net enhancement of action potential generation.

\section{A theory of ganglionic integration}

The experimental results show that convergence typifies the normal synaptic input to secretomotor B neurons. The importance of this observation lies in the minimal nature of the convergence. Because frog B neurons receive so few secondary synapses, their properties can be resolved more easily than can those in homologous mammalian or avian ganglia. This simplicity has enabled us to demonstrate that interactions between minimal numbers of secondary synapses are sufficient to excite sympathetic neurons to threshold. The results thus frame an interesting question about the physiological role of secondary synapses in B neurons and elsewhere. Can a few weak nicotinic synapses contribute in any 
$A$
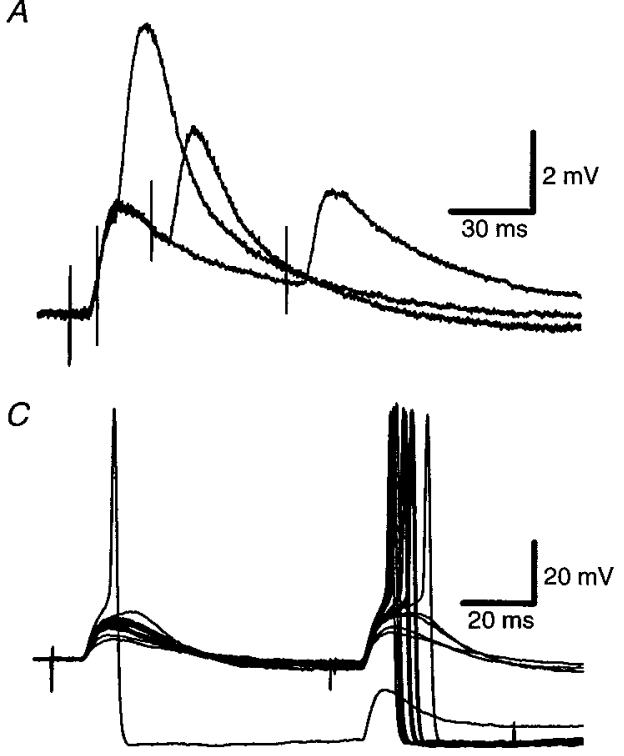

$B$

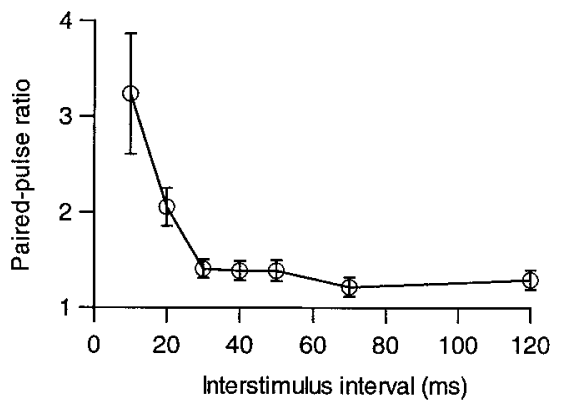

$D$

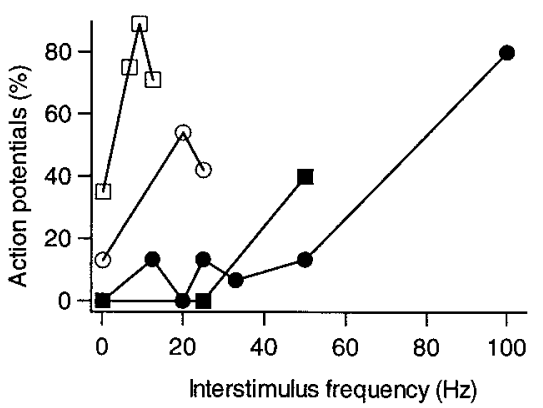

Figure 5. Paired-pulse facilitation of secondary EPSPs can enhance firing. $A$, The time course of facilitation in one neuron, as shown by superimposed records of paired EPSPs, at stimulus intervals of 10,30, and $80 \mathrm{msec}$. Each trace is an average of 6-10 trials after removing responses that evoked action potentials. $B$, The time course of facilitation in grouped data. Paired-pulse ratios [(peak of second response)(peak of first response $)^{-1}$ ] are plotted as a function of stimulus interval (each point represents $6-17$ cells). As in $A$, facilitation of EPSP amplitude is maximal at an interstimulus interval of 10 msec and decays rapidly at longer intervals. $C$, Superimposed trials from a neuron in which stimulation at a two-pulse frequency of $12.5 \mathrm{~Hz}$ increased the proportion of action potentials generated by the second response of the pair. In this case, $R_{\text {leak }}(500 \mathrm{M} \Omega)$ was particularly high, suggesting that the recording represents behavior under conditions of minimal impalement damage. $D$, Cellular variation in the percentage of action potentials generated by the second EPSP at different interstimulus frequencies for four neurons with different $R_{\text {leak }}$ values. Legend: $R_{\text {leak }}=500 \mathrm{M} \Omega(\square), 200 \mathrm{M} \Omega(\square), 130$ $\mathrm{M} \Omega(\bigcirc)$, and $14 \mathrm{M} \Omega(\bullet)$. The interstimulus frequency is the reciprocal of the paired-pulse interval.

meaningful way to the integrated output of sympathetic neurons? The question is deceptively simple. Efforts to answer it in seemingly the most direct manner, by intracellular recording in vivo, have yielded equivocal results because of technical difficulties. In essence, such experiments are hampered by limitations of synapse
$A$

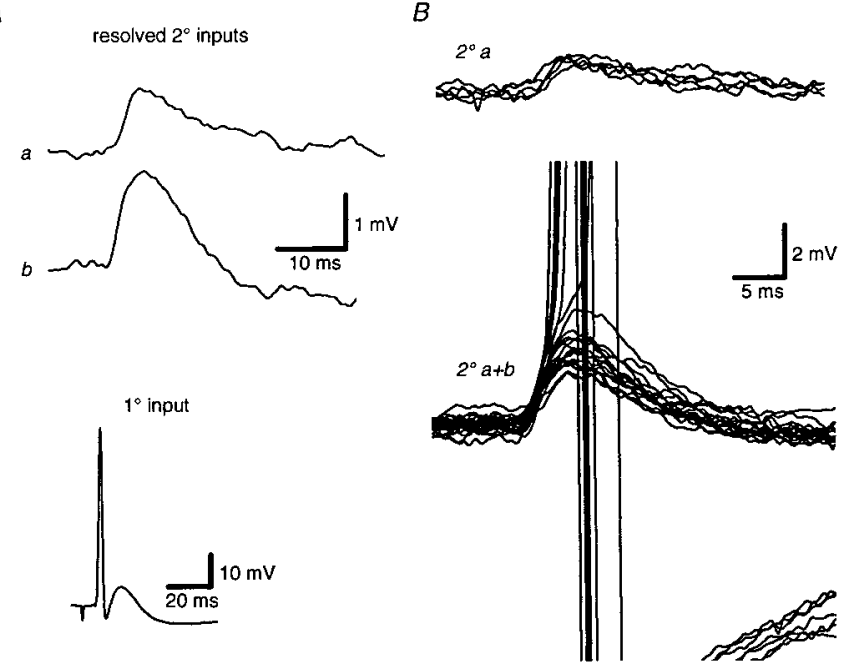

Figure 6. Summation of two secondary EPSPs can enhance firing. $A$, Resolved inputs from a neuron with two secondary synapses $(t o p ; a, b)$ and a primary synapse (bottom) are shown. B, Top, Selective stimulation of the lower threshold input $(A ; a)$ evoked an EPSP that never reached threshold. Bottom, Coactivation of inputs $a+b$ triggered action potentials in 8 of 24 trials. identification, which will be discussed later, and by the fact that anesthesia depresses and disrupts sympathetic behaviors (Jänig, 1995). Taking a different approach, we developed a theoretical model of ganglionic integration that combines our results with the available descriptions of sympathetic activity in vivo.

To construct the model, we first considered the total number of synapses formed by each preganglionic neuron. Organization of the preganglionic neural unit can be inferred from estimates of synaptic divergence and convergence. Preganglionic to postganglionic divergence $(D)$ is 23 in the B system, on the basis of cell counts (Horn et al., 1987; Horn and Stofer, 1988). Convergence, as was noted previously, may follow a general $n+1$ rule in paravertebral ganglia across phylogeny, with each cell receiving $n$ secondary synapses and one primary synapse. The present experimental results indicate $n \geq 1.2$ in the B system. However, little is known about the coupling between primary and secondary synaptic connections. Are all synapses formed by one preganglionic pool of neurons, or do they arise from specialized subsets of preganglionic neurons having distinct patterns of connectivity? We assumed the simplest case, a uniform population of presynaptic neurons in which each cell forms both types of nicotinic synapses. The resulting preganglionic neural unit would then drive 50 ganglion cells $[D \times(n+1)]$ through 23 primary synapses and 27 secondary synapses (Fig. $8 A$ ). The assumption of uniform synaptic connectivity also implies that each ganglionic neuron contributes equally to total postsynaptic activity. In other words the output of the entire circuit can be deduced by considering a single ganglion cell with $n+1$ independent inputs.

Boundary conditions for postsynaptic output can be derived 
$A$

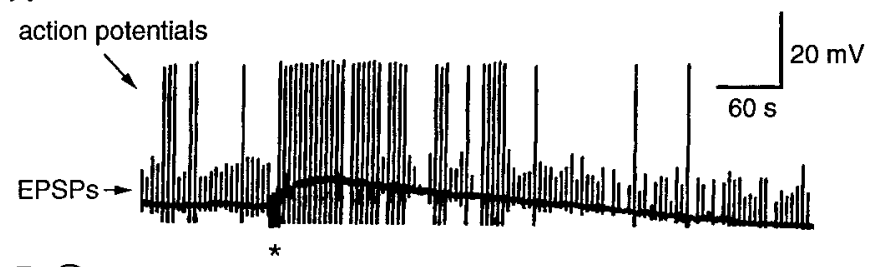

$B$

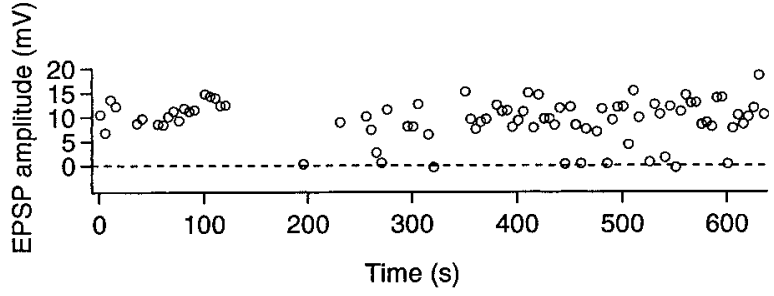

Figure 7. Interaction between secondary EPSPs and the slow peptidergic EPSP can enhance firing. $A$, Chart record of fast EPSPs before and during a slow EPSP generated by stimulation of the preganglionic C pathway $\left(^{*}\right)$. The large fast responses are truncated action potentials. After stimulation of the slow EPSP there was a clear increase in the proportion of secondary EPSPs that triggered action potentials. $B$, Plot of subthreshold EPSP amplitudes showing a slight reduction in the size of nicotinic responses during the slow EPSP and an apparent increase in the failures of fast transmission.

from two idealized extremes of presynaptic activity. The lower boundary is defined by the case in which all preganglionic neurons are synchronously activated at a constant frequency $\left(f_{\text {pre }}\right)$. This condition occurs when preganglionic nerves are repetitively stimulated with supramaximal shocks, a paradigm commonly used in studies of isolated ganglia (Jobling and Horn, 1996; Thorne and Horn, 1997). By definition, synchronous stimulation will always coactivate primary and secondary synapses. Because the primary EPSPs alone are sufficient to fire an action potential in every cell (Shen and Horn, 1995) the entire postsynaptic population will fire at the presynaptic rate $f_{\text {pre }}$. Primary synapses thus set the lower boundary for the ganglionic input-output relation (Fig. $8 B$ ). This relation is invariant and thus hard-wired over the entire physiological range of preganglionic frequencies (i.e., $<20 \mathrm{~Hz}$ ), because of the high safety factor of transmission at the primary synapse (Shen and Horn, 1995). In the entire system, the minimum output of postsynaptic action potentials in a given time interval, $A_{\min }(\Delta t)$, will depend on the number of preganglionic neurons $\left(N_{\text {pre }}\right)$, the preganglionic divergence factor $(D)$, and the preganglionic firing rate $\left(f_{\text {pre }}\right)$ :

$$
A_{\text {min }}(\Delta t)=N_{\text {pre }} D f_{\text {pre }} \Delta t .
$$

The upper boundary for ganglionic output can be derived by considering low-frequency asynchronous preganglionic activity, a condition that better describes physiological behavior in vivo (Ivanoff and Smith, 1995; McLachlan et al., 1997, 1998). In the limit as the average $f_{\text {pre }} \rightarrow 0$, primary and secondary EPSPs will always occur at different times because they arise independently from different neurons whose activity is asynchronous. Because secondary EPSPs evoked at low rates are subthreshold by definition, ganglionic output driven by low-frequency asynchronous activity should normally approximate the lower boundary described by Equation 1. However, if some form of modulation acted to enhance the strength of secondary synapses during asynchronous activity, then the postsynaptic firing rate would increase. Such a possibility is suggested by the observation that the slow peptidergic EPSP can convert fast EPSPs produced by a single secondary synapse from subthreshold to suprathreshold in strength (Fig. 7). In the upper limit for this type of effect, every secondary EPSP in addition to every primary EPSP would trigger an action potential. The upper boundary for ganglionic output $A_{\max }(\Delta t)$ is therefore the sum of all primary and secondary synaptic events (Fig. 8B):

$$
A_{\max }(\Delta t)=(1+n) N_{\text {pre }} D f_{\text {pre }} \Delta t .
$$

At the boundaries defined by Equations 1 and 2, anatomical divergence serves to amplify overall preganglionic activity in the
Figure 8. Schematic depictions of the preganglionic neural unit $(A)$ and its consequences for ganglionic integration $(B)$. $A$, The average preganglionic sympathetic B neuron forms 23 primary nicotinic synapses and 27 secondary nicotinic synapses on $50 \mathrm{~B}$ neurons in paravertebral ganglia 9 and 10. $B$, A model depicts the theoretical input-output relation between preganglionic and ganglionic activity. Preganglionic divergence sets the boundaries of synaptic amplification. The lower boundary for ganglionic output is defined by the divergence of primary nicotinic synapses. The upper boundary is set by the sum of primary and secondary divergence. Synaptic gain within these limits is regulated by preganglionic patterns of activity and by mechanisms that enhance or inhibit the strength of secondary nicotinic synapses. The modulatory mechanism can include presynaptic facilitation of transmitter release and effects mediated by the metabotropic actions of neurotransmitters.

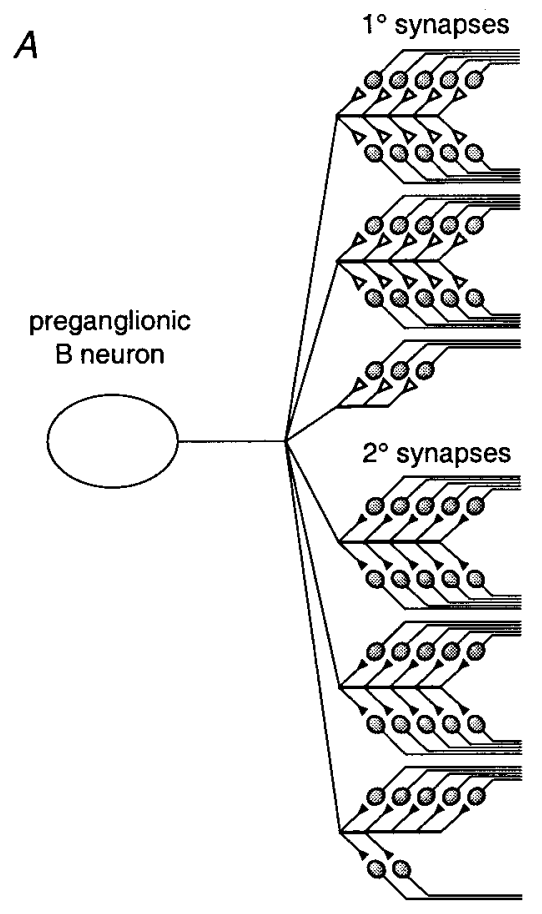

$B$

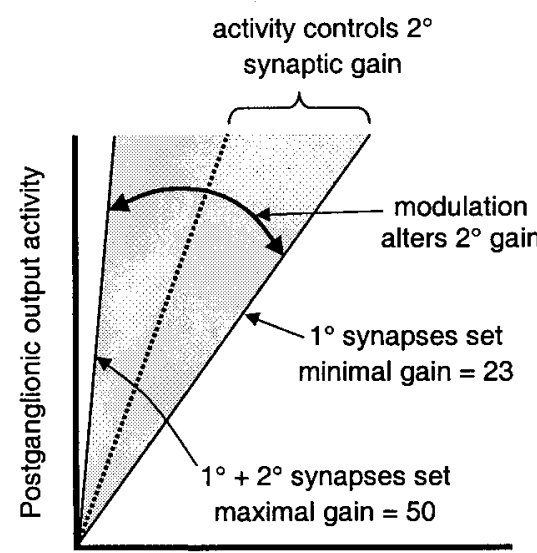

Preganglionic input activity (number of action potentials) 

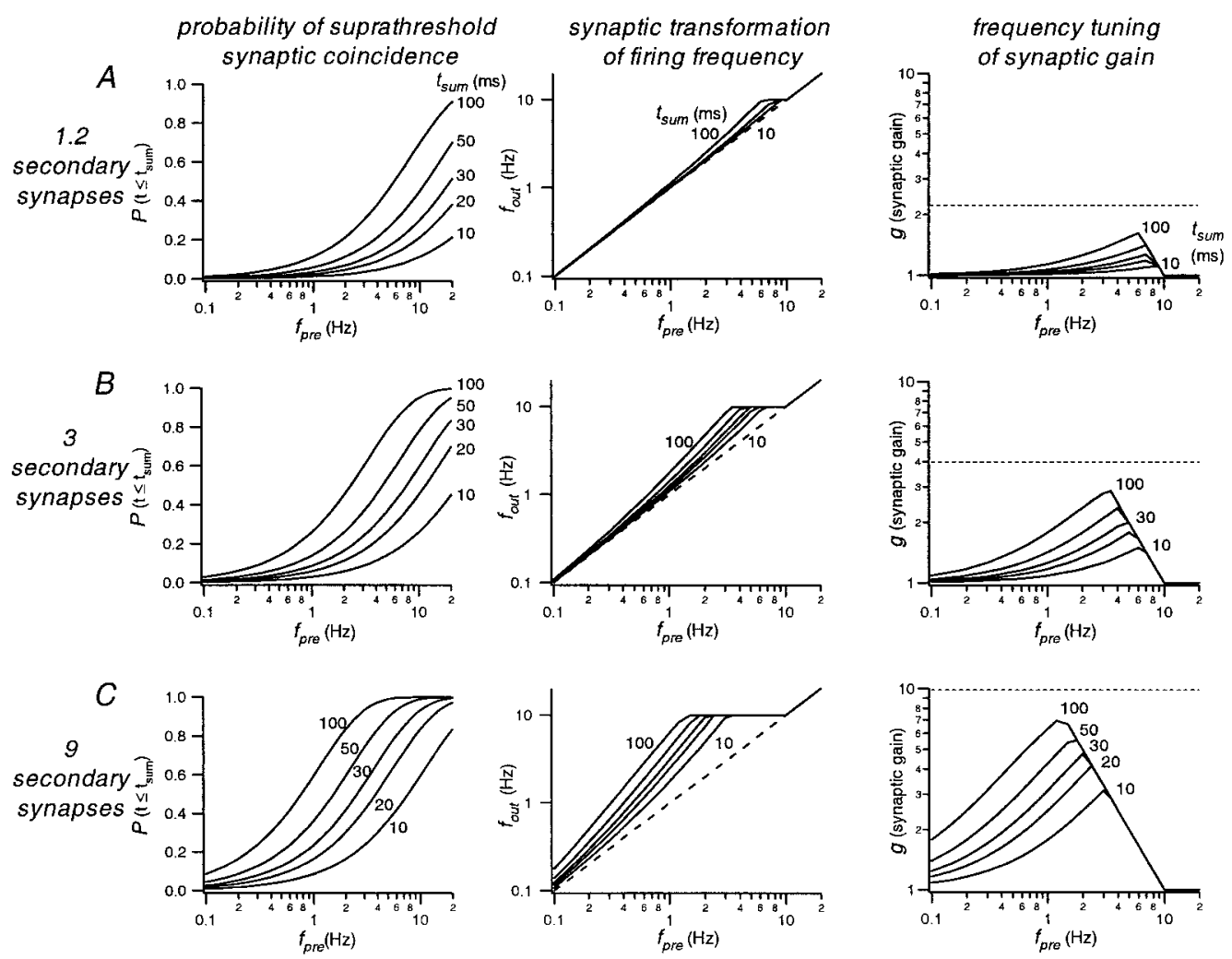

Figure 9. Quantitative predictions based on a stochastic model of synaptic amplification. Calculations were performed for three levels of synaptic convergence. $A$, Convergence of 1.2 secondary synapses and one primary synapse reflects the average observed in our survey of $\mathrm{B}$ neurons. $B$, Convergence of three secondary synapses and one primary synapse mimics the maximal polyinnervation observed in individual B neurons (e.g., Fig. $2 C$ ) and may be more characteristic of vasomotor C neurons (Dodd and Horn, 1983b). $C$, Convergence of nine secondary synapses and one primary synapse approximates the innervation pattern found in the SCG of the rat and guinea pig (Purves et al., 1986). Left Column, Graphs plot Equation 4, the probability that two secondary EPSPs will coincide to trigger an action potential within a window of summation $\left(t_{\text {sum }} ;\right.$ range, $\left.10-100 \mathrm{msec}\right)$, as a function

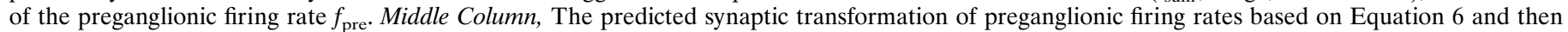
limited so that secondary synapses never can drive action potentials at rates $>10 \mathrm{~Hz}$ is illustrated. Dashed lines in the synaptic transforms depict the lower boundary, in the absence of secondary synaptic activity. Right Column, The cellular synaptic gain relations that were calculated using Equation 7 and the data in the middle column are illustrated. For each set of conditions, synaptic gain is tuned in a nonlinear manner to presynaptic frequencies that lie between 1 and $8 \mathrm{~Hz}$. The tuning shifts to lower $f_{\text {pre }}$ with increases in either $t_{\text {sum }}$ or secondary synaptic convergence $(n)$. Dashed horizontal lines in the three sets of gain relations mark the theoretical upper limit $(n+1)$ for synaptic amplification at each level of convergence.

B system by a factor ranging from 23 to 50 (Fig. $8 B$ ). It should be noted that Equations 1 and 2 also imply that nicotinic EPSPs do not trigger repetitive firing, a condition consistent with our experimental observations.

We next evaluate how the interaction between secondary EPSPs can regulate ganglionic amplification between its boundaries. Temporal interactions arising from presynaptic facilitation (Fig. 5) and postsynaptic summation (Fig. 6) are each capable of initiating action potentials. One can therefore define a window of summation $\left(t_{\text {sum }}\right)$ as the time during which generation of two secondary EPSPs initiates an action potential with $100 \%$ certainty. As part of the model, we also propose that presynaptic facilitation and metabotropic modulation (e.g., slow EPSPs) each regulate the gain of ganglionic transmission by altering $t_{\text {sum }}$ (Fig. $8 B$ ). In this approach, ganglionic amplification can be estimated by calculating the number of coincidences between secondary EPSPs within a given temporal window. The probability of such coincidences can be predicted if one postulates that the generation of EPSPs is a random process, an idea with some experimental basis in mammalian ganglia (McLachlan et al., 1998). For randomly timed synaptic events that occur at an average rate $\lambda$, the intervals between successive EPSPs will be exponentially distributed (Colquhoun, 1971). The probability that two secondary EPSPs occur within an interval $t \leq t_{\text {sum }}$ is:

$$
P\left(t \leq t_{\text {sum }}\right)=1-\exp \left(-\lambda t_{\text {sum }}\right) .
$$

Given the previous assumption of preganglionic uniformity, $\lambda=$ $n f_{\text {pre }}$, and thus:

$$
P\left(t \leq t_{\text {sum }}\right)=1-\exp \left(-n f_{\text {pre }} t_{\text {sum }}\right)
$$

$P\left(t \leq t_{\text {sum }}\right)$ will vary from 0 to 1 with increases in either the average preganglionic firing rate, the window of summation, or the number of converging secondary synapses (Fig. 9, left column; Equation 4). Because precise physiological values for $t_{\text {sum }}$ are unknown, the simulations in Figure 9 explore a range $(10-100$ $\mathrm{msec}$ ) consistent with secondary EPSP duration (e.g., Fig. 1C, 30 $\mathrm{msec}$ ) and the upper temporal limit for suprathreshold facilitation (e.g., Fig. 5C,D, 80-100 msec).

The firing rate of each ganglionic neuron $\left(f_{\text {out }}\right)$ will be approximated by the sum of firing rates attributable to primary synapses $\left(f_{1}\right)$ and secondary synapses $\left(f_{2}\right)$ :

$$
f_{\text {out }} \simeq f_{1}+f_{2} .
$$


Because $f_{1}=f_{\text {pre }}$ and $f_{2}=\lambda P\left(t \leq t_{\text {sum }}\right)=n f_{\text {pre }} P\left(t \leq t_{\text {sum }}\right)$, Equation 5 can be rewritten:

$$
f_{\text {out }} \simeq f_{\text {pre }}\left(1+n P\left(t \leq t_{\text {sum }}\right)\right) .
$$

An oversimplification in Equation 6 is its failure to place an upper limit on postganglionic firing. Clearly, B neurons do not fire at infinitely high frequencies, and postsynaptic action potentials will transiently nullify the interaction between secondary synapses. One solution to this problem would be to expand the model with equations for the refractory period and inhibitory afterpotential after the action potential. Instead, we limited firing rates in the model using a simpler approach. Postsynaptic firing frequencies calculated from Equation 6 were manually adjusted in accord with two constraints: (1) that secondary nicotinic synapses could never drive B cells to fire faster than $10 \mathrm{~Hz}$ and (2) that primary synapses could drive cells at higher frequencies (up to $20 \mathrm{~Hz}$ ). Both assumptions are physiologically plausible (Ivanoff and Smith, 1995; Shen and Horn, 1995), and their precise values are not critical for understanding the model's essential predictions. Figure 9 (middle column) illustrates three examples of the resulting input-output relations for cellular firing rates produced by different degrees of convergence. Several features are worth noting. First, the diagonal (dashed lines; slope of unity) corresponds to the lower boundary attributable to primary nicotinic synapses alone. Second, increases in $t_{\text {sum }}$ act systematically to enhance $f_{\text {out }}$. These relations therefore reproduce the basic input-output relation derived initially from consideration of boundary conditions (Fig. $8 B$ ). Third, the higher the level of secondary synaptic convergence, the larger the increase in $f_{\text {out }}$.

Dividing the ganglionic output frequency by the preganglionic input frequency gives a measure of cellular synaptic gain $(g)$ :

$$
g=f_{\text {out }} / f_{\text {pre }} \text {. }
$$

Plotting $g$ as a function of $f_{\text {pre }}$ (Fig. 9, right column) reveals the theoretical nonlinear tuning of synaptic gain in sympathetic ganglia. In the absence of secondary synapses or with low preganglionic frequencies, $g=1$. Increases in either $t_{\text {sum }}$ or $n$ systematically enhance $g$. Because connections in our model have been assumed to be uniform, the total gain in activity $(G)$ for the entire population of ganglionic B neurons reflects cellular gain and preganglionic divergence $(D)$ :

$$
G=g D
$$

Because $D$ is invariant over time, the dynamic consequences of secondary synapses for ganglionic function can be understood in terms of their effect on cellular gain.

The model predicts that secondary nicotinic synapses enable sympathetic ganglia to function as activity-dependent synaptic amplifiers. Even with only 1.2 secondary synapses and a narrow window of summation $(10 \mathrm{msec})$, preganglionic firing at $7 \mathrm{~Hz}$ is amplified by $10 \%$ (i.e., $g=1.1$ ). Increasing $t_{\text {sum }}$ to $20 \mathrm{msec}$ almost doubles the amplification (19\%), and when $t_{\text {sum }}=50 \mathrm{msec}$, the amplification increases to $41 \%$. These effects grow markedly with higher levels of secondary synaptic convergence, and in addition, $g$ becomes tuned to lower frequencies (Fig. 9). The model therefore predicts that increases in $t_{\text {sum, }}$, whether produced by presynaptic facilitation or slow metabotropic EPSPs, will serve to enhance $g$.

The nonlinear dependence of synaptic gain $(g)$ on the preganglionic firing rate suggests a general mechanism for regulation of the sympathetic outflow $(G)$. Although noisy when observed on a moment-to-moment basis, peripheral sympathetic activity in mammals contains oscillations that can be detected readily by multiunit recording and correlational analysis (McAllen and Malpas, 1997). These oscillations are generated in brainstem circuits, which in turn drive preganglionic activity. Behaviors that enhance postganglionic sympathetic activity are associated with increased coupling to the oscillating activity in the brainstem. We propose that this entrainment of preganglionic activity to oscillations generated by the brainstem serves to regulate the ganglionic amplification of the sympathetic outflow $(G)$ that arises from secondary nicotinic synapses. To illustrate the point, consider a case in which presynaptic activity is asynchronous, $n=3$, and $t_{\text {sum }}$ $=20 \mathrm{msec}$ (Fig. 9B). Under such conditions, the model predicts that cellular gain reaches a maximal value of 1.77 when $f_{\text {pre }}=5$ Hz. Now for simplicity, assume that $f_{\text {pre }}$ remains asynchronous but that it alternates every $0.5 \mathrm{sec}$ between 5 and $1 \mathrm{~Hz}$. This would drive postsynaptic firing $\left(f_{\text {out }}\right)$ at frequencies alternating between 8.88 and $1.17 \mathrm{~Hz}$, with an overall average of $5.02 \mathrm{~Hz}$. By contrast, constant preganglionic firing at $3 \mathrm{~Hz}$ would generate the same number of preganglionic action potentials, but it would drive $f_{\text {out }}$ at only $4.48 \mathrm{~Hz}$. Thus, the crude oscillation generates $12 \%$ more postsynaptic action potentials than does a constant rate of presynaptic firing. Because this effect is a direct consequence of the nonlinear gain of secondary synapses, its magnitude will vary with

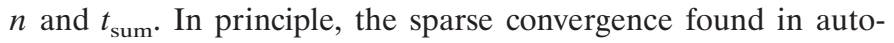
nomic ganglia is very efficient because it enables postsynaptic output to be regulated by the coherence of presynaptic activity (i.e., synchronization), without any absolute requirement for a change in the average presynaptic firing rate.

\section{DISCUSSION}

We have presented evidence that multiple rather than single innervation typifies the normal synaptic input to sympathetic B neurons. Although secondary synapses were much weaker than primary synapses, they could initiate action potentials via several mechanisms. These observations form the basis of a simple theory in which sympathetic ganglia function as synaptic amplifiers. Ganglionic gain is postulated to have a fixed component mediated by primary synapses and a variable component mediated by secondary synapses. The theory also links the seemingly disparate phenomena of non-nicotinic synapses in ganglia, oscillatory activity in the brainstem, and developmental mechanisms that specify the strength and convergence of ganglionic synapses. We propose that their common purpose is to regulate synaptic amplification of the sympathetic outflow in amphibians and other vertebrates.

\section{Ipsilateral origin of secondary synapses}

Secondary synapses on sympathetic B cells were fortuitously discovered when Ivanoff and Smith (1995) recorded natural asynchronous activity in vivo. The extent of subthreshold synaptic activity seen under these conditions was so unexpectedly high that they proposed the existence of a novel contralateral preganglionic pathway, unlike any other found in birds or mammals. How else could one explain that secondary EPSPs had escaped detection during numerous studies of isolated amphibian ganglia, beginning in the 1960s? Our results show that the level of secondary innervation is even greater than first indicated. Because secondary synapses were readily demonstrated in isolated unilateral preparations of ganglia, we would argue that they arise via the conventional uncrossed preganglionic pathway. This interpretation is consistent with previous retrograde tracing, which showed a 
purely ipsilateral preganglionic projection to ganglia 9 and 10 (Horn and Stofer, 1988). One might also ask whether impalement damage could have obscured synaptic convergence in previous studies. This explanation is untenable because secondary EPSPs were detected in most cells, even those with relatively low $\mathrm{V}_{m}$ and $R_{\text {leak }}$ (Fig. 3). Instead it seems likely that secondary EPSPs were overlooked in previous work because their presynaptic stimulus thresholds are very close to those of primary EPSPs and because their relatively small size made them appear insignificant.

\section{Classification of nicotinic synapses by strength}

Defining primary and secondary nicotinic synapses in terms of their ability to initiate action potentials provides a direct link to function. The safety factor for transmission at primary synapses is very high in B neurons, and it remains so over a broad range of stimulus parameters, even in the face of inhibitory modulation (Shen and Horn, 1995). By comparison, secondary EPSPs only reach threshold via fluctuations of release (Fig. 1), facilitation (Fig. 5), summation (Fig. 6), or modulation (Fig. 7). This clear distinction in strength between primary and secondary synapses corresponds to a large difference in quantal content. When compared with previous work (Connor et al., 1983; Shen and Horn, 1996), the present data indicate that $m$ is $10-100$ times larger at primary synapses than at secondary synapses.

Primary and secondary synapses probably have mammalian counterparts, which different authors have classified as "dominant" and "strong" synapses and as "accessory" and "weak" synapses (Skok and Ivanov, 1983; Hirst and McLachlan, 1986; Jänig and McLachlan, 1992; McLachlan et al., 1997, 1998). Although it is tempting to combine these nomenclatures, important distinctions may exist. For example, Skok and Ivanov (1983) maintain that accessory EPSPs in the rabbit SCG reach threshold only via summation, whereas McLachlan et al. (1997, 1998) suggest that weak synapses in the rat SCG contribute little at all to firing. Part of the problem in understanding the precise role of weak/accessory synapses in mammalian ganglia stems from difficulty in identifying individual synapses. In the rat and rabbit SCG, convergence is much greater than that in amphibian ganglia. When recording in vivo one cannot assign individual events to the synapses from which they arise, unless the number of synapses is minimal, as in frog B neurons. It is therefore extremely difficult in mammalian ganglia to determine how many or which synapses contribute to triggering a particular action potential, especially in vivo. Another problem arises because some weak synapses in mammalian ganglia may be strong enough to straddle threshold. This is evident in records of action potentials whose afterpotentials lack any sign of a fast EPSP [see McLachlan et al. (1997), their Fig. 2A]. It is unclear whether such connections should be classified as strong weak synapses or as weak strong synapses. On the basis of our functional criteria, we tentatively favor the former possibility and would simply call them secondary synapses. Further analysis may clarify the role of weak/accessory synapses in mammalian sympathetic ganglia and their relation to secondary synapses in the bullfrog.

\section{Size of the secondary synapse}

Quantal content was $0.31-2.33$ at secondary synapses, on the basis of direct calculation from evoked and asynchronous EPSPs. The distribution of evoked EPSP amplitudes was consistently humplike and symmetric (Fig. 4C,D). This distribution together with a low $m$ indicates that release at secondary synapses is not a Poisson process (McLachlan, 1978). The alternative of a binomial model was only successful in describing the data from one cell (Fig. $4 D$ ), where $p=0.56$ and $N=4$. Assuming every synaptic bouton contains at least one release site, the value of $N$ in this cell suggests that secondary synapses are formed by a handful of boutons. Further support for this interpretation comes from the facilitation data (Fig. 5B). If one assumes that maximal facilitation (i.e., 3.2) arises from an increase in $p$ (Zucker, 1973), then $p<0.3$. Given our estimates of $m$, this would suggest that $N$ is 1-7. At least three possibilities could explain why data from most cells did not fit a standard binomial model. First, $p$ may vary at different secondary release sites, leading to a compound binomial distribution of EPSP amplitudes (McLachlan, 1978; Zucker, 1989). Second, EPSP amplitudes may have been distorted by voltage-activated conductances (Fig. 1C). Third, secondary synapses may be located on the postsynaptic axon at a site removed from the soma, as seen in parasympathetic neurons (Dennis et al., 1971) especially during reinnervation (Roper and Taylor, 1982). These considerations highlight the need for information about the postsynaptic location of secondary synapses and the properties of individual release sites.

\section{Organization of the preganglionic neural unit}

The assumption of uniform synaptic connections implies each preganglionic B neuron innervates 50 sympathetic B neurons (Fig. 8A). This resembles the preganglionic neural unit in the mouse SCG, which contains 64 neurons (Purves et al., 1986). As in previous work on mammalian ganglia, we probably underestimated the true level of convergence and the size of the preganglionic neural unit, because of methodology. When fractionating EPSPs with graded presynaptic stimuli, one only detects secondary axons whose thresholds are lower than that of the primary axon. Secondary EPSPs with high stimulus thresholds are masked by the primary response. The assumption of uniform connections therefore implies a twofold undercounting of secondary synapses. As can be seen from the model (Fig. 9), doubling convergence would markedly increase synaptic gain.

Uniform connections are not essential for synaptic gain. In the rabbit SCG, there may be independent control of accessory and dominant synapses (Skok and Ivanov, 1983). If separate pools of preganglionic neurons form primary and secondary synapses, then only the latter would regulate activity-dependent synaptic gain. This would introduce another layer of control beyond that predicted by our theory.

\section{Brainstem oscillators and ganglionic amplification}

Classical studies by Adrian and colleagues [see McAllen and Malpas (1997)] first demonstrated rhythmic sympathetic activity in mammalian peripheral nerves, with some components phaselocked to the cardiac cycle. The oscillatory activity originates in the rostral medulla, within circuits that drive spinal preganglionic neurons. Progressive activation of cardiovascular pressor reflexes can intensify these oscillations (McAllen and Malpas, 1997). Nonvascular sympathetic cell groups may also be driven by oscillators. Our theory (Fig. 9) predicts that oscillations matched to the tuning of ganglionic gain will amplify the sympathetic outflow. Further elaboration and testing of this hypothesis will require additional attention to the distinctions between functional modalities in the mammalian sympathetic system (Jänig, 1995).

\section{Developmental significance of convergence}

The reason convergence of secondary nicotinic synapses $(n)$ varies within sympathetic ganglia and between species might be related to regulation of ganglionic amplification. Perhaps sympa- 
thetic neurons controlling different peripheral targets require different levels of amplification. It is, for example, more important to maintain tight temporal control over blood pressure than piloerection. Higher open-loop gain in the circuitry controlling vascular resistance would enable blood pressure to be clamped more rapidly and accurately at its physiological set point. Developmental mechanisms that establish ganglionic convergence may regulate in this manner the amplification of activity by functional subsets of sympathetic neurons. Interspecies variation of convergence would allow for scaling of function in animals of different sizes, as suggested by Purves et al. (1986).

\section{Conclusion}

Experiments to analyze a simple example of synaptic convergence have led to a general theory of ganglionic integration, which postulates that sympathetic ganglia are synaptic amplifiers. In the bullfrog B system, we would expect that the effects of ganglionic gain need not be large to be significant. The mucous glands driven by $\mathrm{B}$ cells can respond to single preganglionic stimuli and are half-maximally activated by $0.2 \mathrm{~Hz}$ stimulation (Jobling and Horn, 1996). Because one extra action potential every few seconds can have big effects, ganglionic amplification could appear subtle in vivo.

\section{REFERENCES}

Akasu T, Kojima M, Koketsu K (1983) Luteinizing hormone-releasing hormone modulates nicotinic ACh-receptor sensitivity in amphibian cholinergic transmission. Brain Res 279:347-351.

Allen C, Stevens CF (1994) An evaluation of causes for unreliability of synaptic transmission. Proc Natl Acad Sci USA 91:10380-10383.

Blackman JG, Ginsborg BL, Ray C (1963a) Synaptic transmission in the sympathetic ganglion of the frog. J Physiol (Lond) 167:355-373.

Blackman JG, Ginsborg BL, Ray C (1963b) Spontaneous synaptic activity in sympathetic ganglion cells of the frog. J Physiol (Lond) 167:389-401.

Clements JD, Bekkers JM (1997) Detection of spontaneous synaptic events with an optimally scaled template. Biophys J 73:220-229.

Colquhoun D (1971) Lectures on biostatistics. Oxford: Clarendon.

Connor EA, Levy SM, Parsons RL (1983) Kinetic analysis of atropineinduced alterations in bullfrog ganglionic fast synaptic currents. J Physiol (Lond) 337:137-158.

Dennis MJ, Sargent PB (1978) Multiple innervation of normal and reinnervated parasympathetic neurones in the frog cardiac ganglion. J Physiol (Lond) 281:63-75.

Dennis MJ, Harris AJ, Kuffler SW (1971) Synaptic transmission and its duplication by focally applied acetylcholine in parasympathetic neurons in the heart of the frog. Proc R Soc Lond [Biol] 177:509-539.

Dobrunz LE, Stevens CF (1997) Heterogeneity of release probability, facilitation, and depletion at central synapses. Neuron 18:995-1008.

Dodd J, Horn JP (1983a) A reclassification of B and C neurones in the ninth and tenth paravertebral sympathetic ganglia of the bullfrog. J Physiol (Lond) 334:255-269.

Dodd J, Horn JP (1983b) Muscarinic inhibition of sympathetic C neurones in the bullfrog. J Physiol (Lond) 334:271-291.

Hirst GD, McLachlan EM (1986) Development of dendritic calcium currents in ganglion cells of the rat lower lumbar sympathetic chain. J Physiol (Lond) 377:349-368.

Horn JP, Stofer WD (1988) Spinal origins of preganglionic B and C neurons that innervate paravertebral sympathetic ganglia nine and ten of the bullfrog. J Comp Neurol 268:71-83.

Horn JP, Stofer WD, Fatherazi S (1987) Neuropeptide Y-like immunoreactivity in bullfrog sympathetic ganglia is restricted to C cells. J Neurosci 7:1717-1727.

Horn JP, Fatherazi S, Stofer WD (1988) Differential projections of B and $\mathrm{C}$ sympathetic axons in peripheral nerves of the bullfrog. J Comp Neurol 278:570-580.
Ivanoff AY, Smith PA (1995) In vivo activity of B- and C-neurones in the paravertebral sympathetic ganglia of the bullfrog. J Physiol (Lond) 485:797-815.

Jan YN, Jan LY, Kuffler SW (1979) A peptide as a possible transmitter in sympathetic ganglia of the frog. Proc Natl Acad Sci USA 76:1501-1505.

Jänig W, McLachlan EM (1992) Characteristics of function-specific pathways in the sympathetic nervous system. Trends Neurosci 15:475-481.

Jänig WM (1995) Ganglion transmission in vivo. In: Autonomic ganglia (McLachlan EM, ed), pp 349-395. Luxembourg: Harwood.

Jobling P, Horn JP (1996) In vitro relation between preganglionic sympathetic stimulation and activity of cutaneous glands in the bullfrog. J Physiol (Lond) 494:287-296.

Jones SW (1989) On the resting potential of isolated frog sympathetic neurons. Neuron 3:153-161.

Ko C-P, Roper S (1978) Disorganised and "excessive" reinnervation of frog cardiac ganglia. Nature 274:286-288.

Lang L, Sjöberg E, Skoglund CR (1975) Conductance recording of ionic outflow from frog skin glands during nerve stimulation. Acta Physiol Scand 93:67-76.

Lichtman JW (1980) On the predominantly single innervation of submandibular ganglion cells in the rat. J Physiol (Lond) 302:121-130.

Martin AR, Pilar G (1964) Quantal components of the synaptic potential in the ciliary ganglion of the chick. J Physiol (Lond) 175:1-16.

McAllen RM, Malpas SC (1997) Sympathetic burst activity: characteristics and significance. Clin Exp Pharmacol Physiol 24:791-799.

McLachlan EM (1975) An analysis of the release of acetylcholine from preganglionic nerve terminals. J Physiol (Lond) 245:447-466.

McLachlan EM (1978) The statistics of transmitter release at chemical synapses. Int Rev Physiol 17:49-117.

McLachlan EM, Davies PJ, Häbler HJ, Jamieson J (1997) On-going and reflex synaptic events in rat superior cervical ganglion cells. J Physiol (Lond) 501:165-181.

McLachlan EM, Habler H-J, Jamieson J, Davies PJ (1998) Analysis of the periodicity of synaptic events in neurones in the superior cervical ganglion of anaesthetized rats. J Physiol (Lond) 511:461-478.

Nishi S, Soeda H, Koketsu K (1965) Studies on sympathetic B and C neurons and patterns of preganglionic innervation. J Cell Physiol 66:19-32.

Purves D, Lichtman JW (1985) Geometrical differences among homologous neurons in mammals. Science 228:298-302.

Purves D, Rubin E, Snider WD, Lichtman J (1986) Relation of animal size to convergence, divergence, and neuronal number in peripheral sympathetic pathways. J Neurosci 6:158-163.

Roper S, Taylor B (1982) Reinnervation of denervated parasympathetic neurones in cardiac ganglion from Rana pipiens. J Physiol (Lond) 326:155-171.

Shen WX, Horn JP (1995) A presynaptic mechanism accounts for the differential block of nicotinic synapses on sympathetic B and C neurons by D-tubocurarine. J Neurosci 15:5025-5035.

Shen WX, Horn JP (1996) Presynaptic muscarinic inhibition in bullfrog sympathetic ganglia. J Physiol (Lond) 491:413-421.

Skok VI (1973) Physiology of autonomic ganglia. Tokyo: Igaku Shoin.

Skok VI, Ivanov AY (1983) What is the ongoing activity of sympathetic neurons? J Auton Nerv Syst 7:263-270.

Smith PA (1994) Amphibian sympathetic ganglia: an owner's and operator's manual. Prog Neurobiol 43:439-510.

Stofer WD, Horn JP (1990) Expression of neuropeptide-Y-like immunoreactivity begins after adrenergic differentiation and ganglionic synaptogenesis in developing bullfrog sympathetic neurons. J Neurosci 10:3305-3312.

Thorne R, Horn JP (1997) Role of ganglionic cotransmission in sympathetic control of the isolated bullfrog aorta. J Physiol (Lond) 498:201-214.

Weitsen HA, Weight FF (1977) Synaptic innervation of sympathetic ganglion cells in the bullfrog. Brain Res 128:197-211.

Zucker RS (1973) Changes in the statistics of transmitter release during facilitation. J Physiol (Lond) 229:787-810.

Zucker RS (1989) Short-term synaptic plasticity. Annu Rev Neurosci 12:13-31. 